Nonnegativity Preserving Macro-Element Interpolation of Scattered Data

\author{
Larry L. Schumaker Hendrik Speleers
}

Report TW 543, July 2009

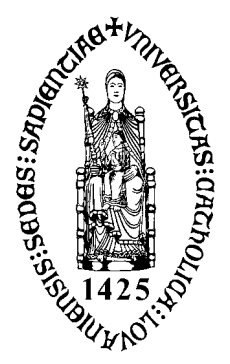

Katholieke Universiteit Leuven Department of Computer Science

Celestijnenlaan 200A - B-3001 Heverlee (Belgium) 


\title{
Nonnegativity Preserving Macro-Element Interpolation of Scattered Data
}

\author{
Larry L. Schumaker Hendrik Speleers \\ Report TW 543, July 2009
}

Department of Computer Science, K.U.Leuven

\begin{abstract}
Nonnegative bivariate interpolants to scattered data are constructed using some $C^{1}$ macro-element spline spaces. The methods are local, and rely on adjusting gradients at the data points to insure nonnegativity of the spline when the original data is nonnegative. More general range-restricted interpolation is also considered.
\end{abstract}

Keywords : spline interpolation, shape preservation, nonnegative surfaces MSC : Primary : 65D07, Secondary : 41A15 


\title{
Nonnegativity Preserving Macro-Element Interpolation of Scattered Data
}

\author{
Larry L. Schumaker ${ }^{1)}$ and Hendrik Speleers ${ }^{2)}$
}

\begin{abstract}
Nonnegative bivariate interpolants to scattered data are constructed using some $C^{1}$ macro-element spline spaces. The methods are local, and rely on adjusting gradients at the data points to insure nonnegativity of the spline when the original data is nonnegative. More general range-restricted interpolation is also considered.
\end{abstract}

Keywords: spline interpolation, shape preservation, nonnegative surfaces

\section{$\S 1$. Introduction}

Interpolating scattered data is a common problem in a wide range of application areas. It is often required that the interpolant preserves known shape properties inherent to the data. Common shape properties are convexity, monotonicity and nonnegativity. In this paper we consider the nonnegativity-preserving interpolation problem.

Problem 1.1. Given a set of scattered data points $\left\{v_{i}:=\left(x_{i}, y_{i}\right)\right\}_{i=1}^{n}$ in a domain $\Omega \subset \mathbb{R}^{2}$ and corresponding nonnegative data values $\left\{f_{i}\right\}_{i=1}^{n}$, find a function $s$ defined on $\Omega$ such that $s\left(x_{i}, y_{i}\right)=f_{i}$ for $i=1, \ldots, n$, and $s(x, y) \geq 0$ for all $(x, y) \in \Omega$.

A standard approach for solving this problem is to work with either polynomial splines $[2,5,6,9,16]$ or rational splines $[1,8,11,12,15]$. These splines are defined on a triangulation with its vertices at the data points. Problem 1.1 is often solved as an optimization problem in order to find a visually pleasing, nonnegative spline that interpolates the data. Such methods are mostly global in nature, i.e., the spline coefficients globally depend on all of the data. Our aim here is to focus on the construction of local and easy to use methods based on standard $C^{1}$ macro-element spline spaces.

The paper is organized as follows. In Sects. 2 and 3 we describe nonnegativitypreserving interpolation methods using the $C^{1}$ Powell-Sabin and Clough-Tocher macro-elements, respectively. We discuss the approximation order of the methods in Sect. 4, and in the following section we explain how to treat certain rangerestricted interpolation problems. Sect. 6 is devoted to some numerical examples. We conclude with some remarks.

1) Department of Mathematics, Vanderbilt University, Nashville, TN 37240, larry.schumaker@vanderbilt.edu .

2) Department of Computer Science, Katholieke Universiteit Leuven, B-3001 Leuven, Belgium, hendrik.speleers@cs.kuleuven. be 


\section{$\S 2$. A Method Based on the $C^{1}$ Powell-Sabin Macro-element}

Suppose $\triangle$ is a triangulation with vertices at the data points $\left\{v_{i}\right\}_{i=1}^{n}$. For each triangle $T \in \triangle$, choose a point $v_{T}$ inside of $T$ and connect it to each of the three vertices of $T$. Connect $v_{T}$ and $v_{\widetilde{T}}$ whenever the triangles $T$ and $\widetilde{T}$ share a common edge. We assume that the points $v_{T}$ are chosen so that these lines cross the common edges. It is well known that this is insured if the $v_{T}$ are taken to be the incenters. In addition, connect the center of each boundary edge to the point $v_{T}$ in the associated triangle. The resulting triangulation $\triangle_{P S}$ is called a Powell-Sabin (PS-) refinement of $\triangle$, see Sect. 4.8.2 of [7]. Let $\mathcal{S}_{2}^{1}\left(\triangle_{P S}\right)$ be the corresponding space of $C^{1}$ piecewise quadratic splines defined on $\triangle_{P S}$. In this section we describe a method for solving Problem 1.1 based on this spline space. We may think of the nonnegative values $\left\{f_{i}\right\}_{i=1}^{n}$ as coming from an unknown nonnegative function $f$ defined on $\Omega$.

\subsection{Hermite Interpolation with $\mathcal{S}_{2}^{1}\left(\triangle_{P S}\right)$}

It is well known (cf. [7]) that for any $\left\{f_{i}, f_{i}^{x}, f_{i}^{y}\right\}_{i=1}^{n}$ there exists a unique (Hermite) interpolating spline $s \in \mathcal{S}_{2}^{1}\left(\triangle_{P S}\right)$ such that

$$
s\left(x_{i}, y_{i}\right)=f_{i}, \quad D_{x} s\left(x_{i}, y_{i}\right)=f_{i}^{x}, \quad D_{y} s\left(x_{i}, y_{i}\right)=f_{i}^{y}, \quad \text { for } i=1, \ldots, n .
$$

In Problem 1.1 we are not given gradients, but there are many methods for estimating them, see Remark 1.

In general, this Hermite interpolant is not guaranteed to be nonnegative, even if the data values and gradients come from a (smooth) nonnegative function $f$. Indeed, at vertices $v_{i}$ where the value $f_{i}$ is near zero, the spline can become negative at some points in a neighborhood of $v_{i}$ if the gradient is too large at $v_{i}$. For an explicit example, see Sect. 6. In the next subsection we show how to adjust the gradients to get a spline which does solve Problem 1.1.

\subsection{Adjusting the gradients}

We now describe a local method for adjusting the gradients in (2.1) so that the corresponding Hermite interpolating spline will provide a solution of Problem 1.1. For each vertex $v_{i}$ of $\triangle$, let $\left\{e_{i j}:=\left\langle v_{i}, v_{i j}\right\rangle\right\}_{j=1}^{n_{i}^{P S}}$ be the set of edges of $\triangle_{P S}$ attached to $v_{i}$. Note that the $v_{i j}$ may be incenters of triangles of $\triangle$, split points on interior edges of $\triangle$, or midpoints of boundary edges of $\triangle$. Suppose the coordinates of $v_{i j}$ are $\left(x_{i j}, y_{i j}\right)$, and let $d_{i j}:=\left(x_{i j}-x_{i}\right) f_{i}^{x}+\left(y_{i j}-y_{i}\right) f_{i}^{y}$.

Algorithm 2.1. For each $i=1, \ldots, n$ :

1) set $\gamma_{i}=1$,

2) for $j=1, \ldots, n_{i}^{P S}$ : if $d_{i j}<0$, set $\gamma_{i}=\min \left\{\gamma_{i},-2 f_{i} / d_{i j}\right\}$,

3) set $\tilde{f}_{i}^{x}=\gamma_{i} f_{i}^{x}$ and $\tilde{f}_{i}^{y}=\gamma_{i} f_{i}^{y}$.

It is easy to see that the $\left\{\gamma_{i}\right\}_{i=1}^{n}$ obtained by this algorithm satisfy $0 \leq \gamma_{i} \leq 1$ for all $i$. 
Theorem 2.2. Given a set of scattered data points $\left\{v_{i}\right\}_{i=1}^{n}$ in $\mathbb{R}^{2}$, let $\triangle$ be a triangulation with vertices at these points, and let $\triangle_{P S}$ be the associated Powell-Sabin refinement. Suppose we are given $\left\{f_{i}, f_{i}^{x}, f_{i}^{y}\right\}_{i=1}^{n}$ with $f_{i} \geq 0$ for $i=1, \ldots, n$. Let $\left\{\tilde{f}_{i}^{x}, \tilde{f}_{i}^{y}\right\}_{i=1}^{n}$ be the adjusted gradients produced by Algorithm 2.1. Then the spline $s \in \mathcal{S}_{2}^{1}\left(\triangle_{P S}\right)$ that interpolates the adjusted data $\left\{f_{i}, \tilde{f}_{i}^{x}, \tilde{f}_{i}^{y}\right\}_{i=1}^{n}$ solves Problem 1.1.

Proof: We examine $\left.s\right|_{T}$ for a fixed macro-triangle $T$ in $\triangle$. Suppose $v_{1}, v_{2}, v_{3}$ are its vertices, and let $c_{1}, \ldots, c_{19}$ be the Bernstein-Bézier (B-) coefficients of $\left.s\right|_{T}$ associated with the domain points in $T$, see Sect. 6.3 of [7] and Fig. 1. Then (cf. equation (6.5) of [7]),

$$
c_{4}=\left[\left(\xi_{13}-x_{1}\right) \tilde{f}_{1}^{x}+\left(\eta_{13}-y_{1}\right) \tilde{f}_{1}^{y}\right] / 2+f_{1},
$$

where $\left(\xi_{13}, \eta_{13}\right)$ are the coordinates of the vertex of $\triangle_{P S}$ lying on the edge $\left\langle v_{1}, v_{2}\right\rangle$. Clearly, the choice of the factor $\gamma_{1}$ implies $c_{4} \geq 0$. A similar argument shows that the coefficients $c_{5}, \ldots, c_{12}$ are also nonnegative. Since all remaining coefficients of $\left.s\right|_{T}$ are convex combinations of these coefficients (cf. equations (6.3) and (6.4) of [7]), we conclude that $\left.s\right|_{T}(x, y) \geq 0$ for all $(x, y) \in T$. Since $T$ was arbitrary, it follows that $s(x, y) \geq 0$ for all $(x, y) \in \Omega$.

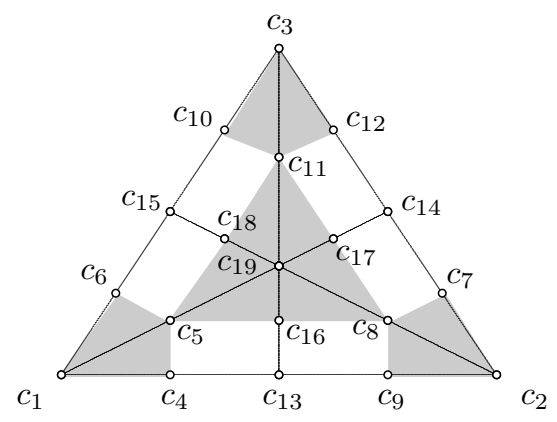

Fig. 1. B-coefficients of the $C^{1}$ PS-macro-element.

For a numerical experiment using this method, see Sect. 6. It is clear from the proof of Theorem 2.2 that we could have constructed a nonnegative spline by simply setting all of the $\gamma_{i}=0$. However, this is not a good strategy as it creates flat spots in the surface at every data point.

\section{$\S 3$. A Method Based on the $C^{1}$ Clough-Tocher Macro-element}

Suppose $\triangle$ is a triangulation with vertices at the data points $\left\{v_{i}\right\}_{i=1}^{n}$. For each triangle $T \in \triangle$, let $v_{T}$ be a point in the interior of $T$, and connect $v_{T}$ to each of the three vertices of $T$. The resulting triangulation $\triangle_{C T}$ is called a Clough-Tocher (CT-) refinement of $\triangle$, see Sect. 4.8.1 of [7] where the split points were chosen to be the barycenters of the triangles of $\triangle$. Here we allow more general split points. In this section we describe a method for solving Problem 1.1 based on the spline space $\mathcal{S}_{3}^{1}\left(\triangle_{C T}\right)$ of $C^{1}$ piecewise cubic splines defined on $\triangle_{C T}$. 


\subsection{Hermite Interpolation with $\mathcal{S}_{3}^{1}\left(\triangle_{C T}\right)$}

For each edge $e:=\left\langle v_{i}, v_{j}\right\rangle$ of $\triangle$, let $\eta_{e}:=\left(v_{i}+v_{j}\right) / 2$ be the midpoint of $e$, and let $D_{u_{e}}$ be the directional derivative associated with the unit vector $u_{e}$ corresponding to rotating $e$ ninety degrees in a counterclockwise direction. Suppose we are given $\left\{f_{i}, f_{i}^{x}, f_{i}^{y}\right\}_{i=1}^{n}$ along with additional real numbers $\left\{g_{e}\right\}_{e \in \mathcal{E}}$, where $\mathcal{E}$ is the set of edges of $\triangle$. Then it is well known (cf. Sect. 6.2 of [7]) that there exists a unique (Hermite) interpolating spline $s \in \mathcal{S}_{3}^{1}\left(\triangle_{C T}\right)$ such that

$$
s\left(x_{i}, y_{i}\right)=f_{i}, \quad D_{x} s\left(x_{i}, y_{i}\right)=f_{i}^{x}, \quad D_{y} s\left(x_{i}, y_{i}\right)=f_{i}^{y}, \quad \text { for } i=1, \ldots, n,
$$

and

$$
D_{u_{e}} s\left(\eta_{e}\right)=g_{e}, \quad \text { for all } e \in \mathcal{E} .
$$

In Problem 1.1 we are not generally given the gradient values $\left.\left\{f_{i}^{x}, f_{i}^{y}\right)\right\}_{i=1}^{n}$ or the cross derivative values $\left\{g_{e}\right\}_{e \in \mathcal{E}}$, but there are many methods for estimating them, see Remarks 1 and 2 .

In general, the above Hermite interpolant is not guaranteed to be nonnegative, even if the data values and gradients come from a (smooth) nonnegative function $f$. As in the Powell-Sabin case, at vertices $v_{i}$ where the value $f_{i}$ is near zero, the spline can become negative at some points in a neighborhood of $v_{i}$ if the gradient is too large at $v_{i}$. For an explicit example, see Sect. 6. In Sects. 3.4 and 3.5 we show how to adjust the gradients $\left\{f_{i}^{x}, f_{i}^{y}\right\}_{i=1}^{n}$ and the values $\left\{g_{e}\right\}_{e \in \mathcal{E}}$ to get a spline which solves Problem 1.1. First we need to derive some sufficient conditions for a $C^{1}$ CT-macro-element patch to be nonnegative.

\subsection{Nonnegativity of a cubic CT-macro-element patch}

Given a triangle $T:=\left\langle v_{1}, v_{2}, v_{3}\right\rangle$, let $T_{C T}$ be the CT-refinement obtained by splitting $T$ into three subtriangles by inserting a vertex $v_{T}:=\alpha_{1}^{T} v_{1}+\alpha_{2}^{T} v_{2}+\alpha_{3}^{T} v_{3}$, where the $\alpha_{i}^{T}>0$ and $\alpha_{1}^{T}+\alpha_{2}^{T}+\alpha_{3}^{T}=1$. It is well known (cf. Sect. 6.2 of [7]) that $\operatorname{dim} \mathcal{S}_{3}^{1}\left(T_{C T}\right)=12$. Now fix $s \in \mathcal{S}_{3}^{1}\left(T_{C T}\right)$, and suppose its B-coefficients are $\left\{c_{i}\right\}_{i=1}^{19}$ as shown in Fig. 2, where we orient $T$ so that $c_{1}$ is the coefficient associated with $v_{1}$. Then as shown in Theorem 6.5 in [7], $s$ is uniquely determined by the coefficients $\left\{c_{i}\right\}_{i=1}^{12}$, and the remaining coefficients are determined (from $C^{1}$ smoothness conditions) by the formulae

$$
\begin{aligned}
& c_{13}=\alpha_{1}^{T} c_{1}+\alpha_{2}^{T} c_{4}+\alpha_{3}^{T} c_{9}, \\
& c_{14}=\alpha_{1}^{T} c_{5}+\alpha_{2}^{T} c_{2}+\alpha_{3}^{T} c_{6}, \\
& c_{15}=\alpha_{1}^{T} c_{8}+\alpha_{2}^{T} c_{7}+\alpha_{3}^{T} c_{3}, \\
& c_{16}=\alpha_{1}^{T} c_{13}+\alpha_{2}^{T} c_{10}+\alpha_{3}^{T} c_{12}, \\
& c_{17}=\alpha_{1}^{T} c_{10}+\alpha_{2}^{T} c_{14}+\alpha_{3}^{T} c_{11}, \\
& c_{18}=\alpha_{1}^{T} c_{12}+\alpha_{2}^{T} c_{11}+\alpha_{3}^{T} c_{15}, \\
& c_{19}=\alpha_{1}^{T} c_{16}+\alpha_{2}^{T} c_{17}+\alpha_{3}^{T} c_{18} .
\end{aligned}
$$




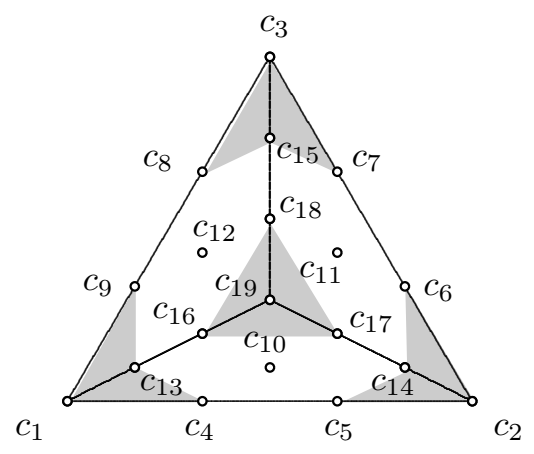

Fig. 2. B-coefficients of a CT-macro-element.

In this section we discuss conditions on the coefficients of $s$ that guarantee that it is nonnegative on $T$. Since $c_{i}=s\left(v_{i}\right)$ for $i=1,2,3$, it is clear that one necessary condition is that

$$
m:=\min \left\{c_{1}, c_{2}, c_{3}\right\} \geq 0
$$

Finding a complete set of necessary and sufficient conditions seems to be a complicated problem, and has not even been solved for a single cubic polynomial defined on a triangle, see Remark 3. In view of the formulae (3.3) and the nature of the B-form representation, the conditions $c_{i} \geq 0, i=1, \ldots, 12$, are sufficient. Inspired by ideas in $[6]$, we now derive a weaker set of sufficient conditions. Fix

$$
a \geq \max \left\{1, \frac{1-\alpha_{1}^{T}}{3 \alpha_{1}^{T}}, \frac{1-\alpha_{2}^{T}}{3 \alpha_{2}^{T}}, \frac{1-\alpha_{3}^{T}}{3 \alpha_{3}^{T}}\right\}
$$

where we recall that $\left(\alpha_{1}^{T}, \alpha_{2}^{T}, \alpha_{3}^{T}\right)$ are the barycentric coordinates of $v_{T}$ relative to triangle $T$.

Lemma 3.1. Let $\left\{c_{i}\right\}_{i=1}^{12}$ be given numbers such that (3.4) holds. Suppose in addition that

$$
c_{i} \geq-\frac{m}{3 a}, \quad 4 \leq i \leq 9
$$

where a satisfies (3.5), and let

$$
\begin{aligned}
& c_{10} \geq-\frac{1}{2} \min \left\{\frac{\alpha_{1}^{T}}{\alpha_{2}^{T}}, \frac{\alpha_{2}^{T}}{\alpha_{1}^{T}}\right\} \min \left\{c_{13}, c_{14}\right\}, \\
& c_{11} \geq-\frac{1}{2} \min \left\{\frac{\alpha_{2}^{T}}{\alpha_{3}^{T}}, \frac{\alpha_{3}^{T}}{\alpha_{2}^{T}}\right\} \min \left\{c_{14}, c_{15}\right\}, \\
& c_{12} \geq-\frac{1}{2} \min \left\{\frac{\alpha_{1}^{T}}{\alpha_{3}^{T}}, \frac{\alpha_{3}^{T}}{\alpha_{1}^{T}}\right\} \min \left\{c_{13}, c_{15}\right\} .
\end{aligned}
$$


Let $\left\{c_{i}\right\}_{i=13}^{19}$ be given by (3.3). Then the corresponding spline $s \in \mathcal{S}_{3}^{1}\left(\triangle_{C T}\right)$ is nonnegative on $T$.

Proof: Combining (3.4)-(3.6), we see that

$$
c_{13}=\alpha_{1}^{T} c_{1}+\alpha_{2}^{T} c_{4}+\alpha_{3}^{T} c_{9} \geq m\left(\alpha_{1}^{T}-\frac{\alpha_{2}^{T}}{3 a}-\frac{\alpha_{3}^{T}}{3 a}\right)=m \alpha_{1}^{T}\left(1-\frac{1-\alpha_{1}^{T}}{3 a \alpha_{1}^{T}}\right) \geq 0 .
$$

Similarly, $c_{14}, c_{15} \geq 0$. Now inserting (3.7) and (3.9) in (3.3) gives

$$
c_{16}=\alpha_{1}^{T} c_{13}+\alpha_{2}^{T} c_{10}+\alpha_{3}^{T} c_{12} \geq c_{13} \alpha_{1}^{T}\left(1-\frac{1}{2}-\frac{1}{2}\right) \geq 0 .
$$

A similar computation shows that $c_{17}, c_{18} \geq 0$. It then follows from (3.3) that $c_{19} \geq 0$.

We now focus on the micro-triangle $t:=\left\langle v_{1}, v_{2}, v_{T}\right\rangle$. Following an idea from [6], fix $0 \leq \xi \leq 1$, and consider the restriction $p_{\xi}$ of $s$ to the line from $v_{T}$ to the point $v_{\xi}:=(1-\xi) v_{1}+\xi v_{2}$. Then $p_{\xi}$ is a univariate cubic polynomial which can be written in Bernstein-Bézier form as

$$
p_{\xi}(\tau)=d_{0}(1-\tau)^{3}+3 d_{1} \tau(1-\tau)^{2}+3 d_{2} \tau^{2}(1-\tau)+d_{3} \tau^{3}, \quad 0 \leq \tau \leq 1,
$$

where $d_{0}=c_{19}$ and

$$
\begin{aligned}
& d_{1}:=c_{16}(1-\xi)+c_{17} \xi \\
& d_{2}:=c_{13}(1-\xi)^{2}+2 c_{10} \xi(1-\xi)+c_{14} \xi^{2} \\
& d_{3}:=c_{1}(1-\xi)^{3}+3 c_{4} \xi(1-\xi)^{2}+3 c_{5} \xi^{2}(1-\xi)+c_{2}(1-\xi)^{3} .
\end{aligned}
$$

It is known (cf. $[3,17])$ that a cubic polynomial of the form $(3.11)$ is nonnegative for $0 \leq \tau \leq 1$ if and only if

a) $d_{0}, d_{1}, d_{2}, d_{3} \geq 0$,

or

b) $d_{0}, d_{3} \geq 0$ and $4\left(d_{0} d_{2}^{3}+d_{3} d_{1}^{3}\right)+d_{0}^{2} d_{3}^{2}-6 d_{0} d_{1} d_{2} d_{3}-3 d_{1}^{2} d_{2}^{2} \geq 0$.

We now verify that a) holds. First, since $c_{16}, c_{17} \geq 0$, it follows that $d_{1} \geq 0$. It is known (cf. $[10,17]$ ) that a necessary and sufficient condition for the quadratic polynomial $d_{2}$ to be nonnegative for $0 \leq \xi \leq 1$ is that $c_{13}, c_{14} \geq 0$ and $c_{10} \geq$ $-\sqrt{c_{13} c_{14}}$. In view of (3.10) and (3.7), it follows that $d_{2} \geq 0$. To show that $d_{3} \geq 0$, we first verify that the analog of b) holds with the coefficients $c_{1}=c_{2}=m$ and $c_{4}=c_{5}=-m /(3 a)$ and $a \geq 1$. With these coefficients we have

$$
4\left(c_{1} c_{5}^{3}+c_{2} c_{4}^{3}\right)+c_{1}^{2} c_{2}^{2}-6 c_{1} c_{4} c_{5} c_{2}-3 c_{4}^{2} c_{5}^{2}=\frac{m^{4}}{27 a^{4}}\left(27 a^{4}-18 a^{2}-8 a-1\right) \geq 0 .
$$

But then the polynomial $d_{3}$ is also nonnegative for any choice of $c_{1}, c_{2} \geq m$ and $c_{4}, c_{5} \geq-m /(3 a)$. 
We conclude that $p_{\xi}(\tau) \geq 0$ for all $0 \leq \tau \leq 1$. Since this holds for all $0 \leq \xi \leq 1$, we conclude that $s$ is nonnegative at all points in the triangle $\left\langle v_{1}, v_{2}, v_{T}\right\rangle$. A similar argument shows that it is nonnegative at all points in the other two subtriangles of $T$.

Based on this lemma, we can now develop an algorithm for solving Problem 1.1 with a spline $s \in \mathcal{S}_{3}^{1}\left(\triangle_{C T}\right)$. The algorithm proceeds as follows:

1) estimate the gradients at the data points $\left\{v_{i}\right\}_{i=1}^{n}$,

2 ) estimate the normal cross-derivatives at midpoints of each edge $e$ of $\triangle$,

3 ) adjust the gradients so that (3.6) is satisfied for every triangle $T \in \triangle$. Compute a spline $s \in \mathcal{S}_{3}^{1}\left(\triangle_{C T}\right)$ interpolating this adjusted data,

4) further adjust the coefficients of $s$ so that the remaining conditions of Lemma 3.1 are satisfied for every triangle $T$.

For references to some methods for steps 1) and 2), see Remarks 1 and 2. We discuss steps 3) and 4) in the following two sections.

\subsection{Adjusting the gradients}

As a first step towards constructing a spline $s \in \mathcal{S}_{3}^{1}\left(\triangle_{C T}\right)$ solving Problem 1.1, we now describe a local method for adjusting the gradients $\left\{f_{i}^{x}, f_{i}^{y}\right\}_{i=1}^{n}$ so that the Bcoefficients of the corresponding Hermite interpolating spline satisfy the inequalities (3.6) of Lemma 3.1 for every triangle $T \in \triangle$,

For each vertex $v_{i}$ of $\triangle$, let $\left\{e_{i j}:=\left\langle v_{i}, v_{i j}\right\rangle\right\}_{j=1}^{n_{i}}$ be the set of edges of $\triangle$ attached to $v_{i}$. Suppose the coordinates of $v_{i j}$ are $\left(x_{i j}, y_{i j}\right)$, and let $d_{i j}:=\left(x_{i j}-\right.$ $\left.x_{i}\right) f_{i}^{x}+\left(y_{i j}-y_{i}\right) f_{i}^{y}$. Let $m_{i j}:=\min \left\{f_{k}: f_{k}\right.$ is a data value associated with a vertex of a triangle containing $\left.e_{i j}\right\}$. Let

$$
a_{i j} \geq \max _{T \in \mathcal{T}_{i j}} \max \left\{1, \frac{1-\alpha_{1}^{T}}{3 \alpha_{1}^{T}}, \frac{1-\alpha_{2}^{T}}{3 \alpha_{2}^{T}}, \frac{1-\alpha_{3}^{T}}{3 \alpha_{3}^{T}}\right\},
$$

where $\mathcal{T}_{i j}$ is the set of all triangles sharing the edge $e_{i j}$.

Algorithm 3.2. For each $i=1, \ldots, n$ :

1) set $\gamma_{i}=1$,

2) for $j=1, \ldots, n_{i}$ : if $d_{i j}<0$, set $\gamma_{i}=\min \left\{\gamma_{i},\left(-3 f_{i}-m_{i j} / a_{i j}\right) / d_{i j}\right\}$,

3) set $\tilde{f}_{i}^{x}=\gamma_{i} f_{i}^{x}$ and $\tilde{f}_{i}^{y}=\gamma_{i} f_{i}^{y}$. all $i$.

It is clear that the $\left\{\gamma_{i}\right\}_{i=1}^{n}$ produced by this algorithm satisfy $0 \leq \gamma_{i} \leq 1$ for

Lemma 3.3. Given a set of scattered data points $\left\{v_{i}\right\}_{i=1}^{n}$ in $\mathbb{R}^{2}$, let $\triangle$ be a triangulation with vertices at these points, and let $\triangle_{C T}$ be the associated Clough-Tocher refinement. Suppose we are given $\left\{f_{i}, f_{i}^{x}, f_{i}^{y}\right\}_{i=1}^{n}$ with $f_{i} \geq 0$ for $i=1, \ldots, n$, along with additional values $\left\{g_{e}\right\}_{e \in \mathcal{E}}$, where $\mathcal{E}$ is the set of edges of $\triangle$. Let $\left\{\tilde{f}_{i}^{x}, \tilde{f}_{i}^{y}\right\}_{i=1}^{n}$ be the adjusted gradients produced by Algorithm 3.2, and let $s$ be the spline in 
$\mathcal{S}_{3}^{1}\left(\triangle_{C T}\right)$ that interpolates as in (3.1) to this adjusted data. Then the B-coefficients of $s$ satisfy (3.6) for every triangle $T \in \triangle$.

Proof: Fix $T$ in $\triangle$. We may suppose $v_{1}, v_{2}, v_{3}$ are its vertices, and let $c_{1}, \ldots, c_{19}$ be the B-coefficients of $\left.s\right|_{T}$ associated with the domain points in $T$ as in Fig. 2. Then (cf. equation (6.2) of [7]),

$$
c_{4}=\left[\left(x_{2}-x_{1}\right) \tilde{f}_{1}^{x}+\left(y_{2}-y_{1}\right) \tilde{f}_{1}^{y}\right] / 3+f_{1}
$$

and the choice of $\gamma_{i}$ insures that $c_{4}$ satisfies (3.6) with $m:=\min \left\{f_{1}, f_{2}, f_{3}\right\} . \mathrm{A}$ similar argument shows that the coefficients $c_{5}, \ldots, c_{9}$ also satisfy (3.6).

\subsection{Adjusting the coefficients of $s$}

The spline $s$ in Lemma 3.3 is not necessarily a solution of Problem 1.1, since if the given cross derivatives $\left\{g_{e}\right\}_{e \in \mathcal{E}}$ are too large for certain edges, we may get negative values for $s$ near these edges. While it is possible to adjust the $\left\{g_{e}\right\}_{e \in \mathcal{E}}$ in the same way that we adjusted gradients in the previous section, in this section we instead show how to adjust the B-coefficients of $s$ directly to insure that conditions (3.7)(3.9) hold for each triangle $T$. This must be done with care when two triangles share an edge since we need to make sure that all $C^{1}$ conditions across the edge are satisfied.

For each boundary edge $e$ of $\triangle$, there is one triangle $T_{e}:=\left\langle v_{1}^{e}, v_{2}^{e}, v_{3}^{e}\right\rangle$ of $\triangle$ containing $e$. We denote the corresponding micro-triangle which contains $e$ by $t_{e}:=\left\langle v_{1}^{e}, v_{2}^{e}, v_{T_{e}}\right\rangle$, where $v_{T_{e}}$ is the split point in $T_{e}$. If $e$ is an interior edge of $\triangle$, there is a second triangle $\hat{T}_{e}:=\left\langle v_{2}^{e}, v_{1}^{e}, \hat{v}_{3}^{e}\right\rangle$ of $\triangle$ containing $e$. Let $v_{\hat{T}_{e}}$ be the split point in $\hat{T}_{e}$, and let $\hat{t}_{e}:=\left\langle v_{2}^{e}, v_{1}^{e}, v_{\hat{T}_{e}}\right\rangle$ be the corresponding micro-triangle containing $e$. Let $\left(\alpha_{1}^{e}, \alpha_{2}^{e}, \alpha_{3}^{e}\right)$ be the barycentric coordinates of $v_{T_{e}}$ relative to $T_{e}$, and let $\left(\hat{\alpha}_{2}^{e}, \hat{\alpha}_{1}^{e}, \hat{\alpha}_{3}^{e}\right)$ be the barycentric coordinates of $v_{\hat{T}_{e}}$ relative to $\hat{T}_{e}$. Suppose $\left(\beta_{1}^{e}, \beta_{2}^{e}, \beta_{3}^{e}\right)$ are the barycentric coordinates of $v_{\hat{T}_{e}}$ relative to $t_{e}$, i.e., $v_{\hat{T}_{e}}=\beta_{1}^{e} v_{1}^{e}+\beta_{2}^{e} v_{2}^{e}+\beta_{3}^{e} v_{T_{e}}$. We note that $\beta_{3}^{e}<0$. We write $\left\{c_{i}^{e}\right\}_{i=1}^{19}$ and $\left\{\hat{c}_{i}^{e}\right\}_{i=1}^{19}$ for the coefficients of $\left.s\right|_{T_{e}}$ and $\left.s\right|_{\hat{T}_{e}}$, where the orientation is chosen so that $c_{10}^{e}$ and $\hat{c}_{10}^{e}$ are next to the edge $e$.

\section{Algorithm 3.4.}

1) For each boundary edge $e$ of $\triangle$ : if necessary, increase $c_{10}^{e}$ to satisfy (3.7).

2) For each interior edge $e$ of $\triangle$ : if necessary, increase $c_{10}^{e}$ so that it satisfies (3.7). Then compute the corresponding coefficient $\hat{c}_{10}^{e}$ from the $C^{1}$ smoothness condition across $e$, i.e., set $\hat{c}_{10}^{e}=\beta_{1}^{e} c_{4}^{e}+\beta_{2}^{e} c_{5}^{e}+\beta_{3}^{e} c_{10}^{e}$. If necessary, increase $\hat{c}_{10}^{e}$ so that it satisfies the analog of (3.7). Then recompute the coefficient $c_{10}^{e}$ from the $C^{1}$ smoothness condition across $e$.

We shall show in Theorem 3.6 below that after applying both Algorithms 3.2 and 3.4, the resulting spline will be a solution of Problem 1.1. But first we need 
to discuss whether step 2) of Algorithm 3.4 is well-defined. For this to happen, we need

$$
\begin{aligned}
I:=\frac{1}{2} \min \left\{\frac{\hat{\alpha}_{1}^{e}}{\hat{\alpha}_{2}^{e}},\right. & \left.\frac{\hat{\alpha}_{2}^{e}}{\hat{\alpha}_{1}^{e}}\right\} \min \left\{\hat{c}_{13}^{e}, \hat{c}_{14}^{e}\right\}+\beta_{1}^{e} c_{4}^{e}+\beta_{2}^{e} c_{5}^{e} \\
& -\frac{\beta_{3}^{e}}{2} \min \left\{\frac{\alpha_{1}^{e}}{\alpha_{2}^{e}}, \frac{\alpha_{2}^{e}}{\alpha_{1}^{e}}\right\} \min \left\{c_{13}^{e}, c_{14}^{e}\right\} \geq 0
\end{aligned}
$$

The interval $[0, I]$ is a shifted version of the intervals to which $c_{10}^{e}$ and $\hat{c}_{10}^{e}$ are restricted by inequality (3.7) and by the $C^{1}$ smoothness condition across edge $e$.

Lemma 3.5. Suppose $T_{e}$ and $\hat{T}_{e}$ are neighboring triangles of $\triangle_{C T}$ sharing an edge $e$, and suppose $\beta_{1}^{e}, \beta_{2}^{e} \geq 0$. Suppose we choose a so that (3.5) holds for both $T_{e}$ and $\hat{T}_{e}$, and that in addition

$$
a \geq \frac{2+A(1-B)}{3 A B},
$$

where

$$
A:=\min \left\{\frac{\alpha_{1}^{e}}{\alpha_{2}^{e}}, \frac{\alpha_{2}^{e}}{\alpha_{1}^{e}}, \frac{\hat{\alpha}_{1}^{e}}{\hat{\alpha}_{2}^{e}}, \frac{\hat{\alpha}_{2}^{e}}{\hat{\alpha}_{1}^{e}}\right\}, \quad \text { and } \quad B:=\min \left\{\alpha_{1}^{e}, \alpha_{2}^{e}, \hat{\alpha}_{1}^{e}, \hat{\alpha}_{2}^{e}\right\}
$$

Suppose $\left\{c_{i}\right\}_{i=1}^{9}$ and $\left\{\hat{c}_{i}\right\}_{i=1}^{9}$ satisfy (3.4) and (3.6). Then (3.16) is satisfied.

Proof: Combining (3.6) with (3.10), we can bound $I$ from below as

$$
\begin{aligned}
I & \geq \frac{m}{2}\left(1-\beta_{3}^{e}\right) A\left[\left(1+\frac{1}{3 a}\right) B-\frac{1}{3 a}\right]-\left(\beta_{1}^{e}+\beta_{2}^{e}\right) \frac{m}{3 a} \\
& =m\left(1-\beta_{3}^{e}\right)\left[\left(\frac{A}{2}(B-1)-1\right) \frac{1}{3 a}+\frac{A B}{2}\right],
\end{aligned}
$$

which is nonnegative by the choice of $a$.

The condition on $\beta_{1}^{e}$ and $\beta_{2}^{e}$ in Lemma 3.5 is equivalent to requiring that the line joining the split points $v_{T_{e}}$ and $v_{\hat{T}_{e}}$ inside $T_{e}$ and $\hat{T}_{e}$ must intersect the line segment $e$. This is equivalent to the restriction on the split points used in defining Powell-Sabin refinements. Lemma 4.19 in [7] insures that this will be the case if we take the split points to be the incenters. It is not hard to construct an example showing that if this condition is not satisfied, then the quantity $I$ in (3.16) cannot be made nonnegative no matter how we choose $a$, see Remark 6 . The following theorem is now obvious.

Theorem 3.6. Given a set of scattered data points $\left\{v_{i}\right\}_{i=1}^{n}$ in $\mathbb{R}^{2}$, let $\triangle$ be a triangulation with vertices at these points, and let $\triangle_{C T}$ be the associated CloughTocher refinement such that the lines joining split points of adjoining triangles of $\triangle$ intersect their common edge. Suppose we are given $\left\{f_{i}, f_{i}^{x}, f_{i}^{y}\right\}_{i=1}^{n}$ with $f_{i} \geq 0$ for $i=1, \ldots, n$, along with additional values $\left\{g_{e}\right\}_{e \in \mathcal{E}}$, where $\mathcal{E}$ is the set of edges 
of $\triangle$. Suppose for each edge of $\triangle$, we choose $a_{i j}$ to satisfy (3.15), and also (3.17) if it is an interior edge. Suppose the gradients at the data points are adjusted by applying Algorithm 3.2. Let $s \in \mathcal{S}_{3}^{1}\left(\triangle_{C T}\right)$ be the corresponding interpolating spline in Lemma 3.3, and suppose its B-coefficients are further adjusted by Algorithm 3.4. Then the resulting spline solves Problem 1.1.

For many triangulations $\triangle$, the choice of barycenters for the split points results in a refined triangulation $\triangle_{C T}$ with $\beta_{1}^{e}, \beta_{2}^{e} \geq 0$ for all interior edges. In this case we can choose all $a_{i j}=8 / 3$ in this theorem.

\section{$\S 4$. Approximation Order}

In this section we provide error bounds for our interpolation methods measured in the maximum norm. To measure smoothness of the interpolated function $f$, we use the standard Sobolev semi-norms

$$
|f|_{k}=\max _{\nu+\mu=k}\left\|D_{x}^{\nu} D_{y}^{\mu} f\right\|
$$

where in this section $\|\cdot\|$ always refers to the maximum norm on $\Omega$. We denote the diameter of triangulation $\triangle$ by $|\triangle|$. For comparison purposes, we also quote results giving error bounds for unrestricted Hermite interpolation with $C^{1}$ Powell-Sabin and Clough-Tocher macro-element spaces.

\subsection{The Powell-Sabin Case}

The following result gives an error bound for the unrestricted Powell-Sabin interpolant $\mathcal{I}_{P S}$, see Theorem 6.12 in [7].

Theorem 4.1. For every $f \in C^{k+1}(\Omega)$ with $0 \leq k \leq 2$,

$$
\left\|D_{x}^{\alpha} D_{y}^{\beta}\left(f-\mathcal{I}_{P S} f\right)\right\| \leq K_{1}|\triangle|^{k+1-\alpha-\beta}|f|_{k+1},
$$

for all $0 \leq \alpha+\beta \leq k$. If $\Omega$ is convex, then the constant $K_{1}$ depends only on the smallest angle in $\triangle$. If $\Omega$ is not convex, then $K_{1}$ also depends on the Lipschitz constant of the boundary of $\Omega$.

We now establish an analogous error bound for our nonnegative Powell-Sabin interpolant $\mathcal{I}_{P S}^{+}$. Given a triangulation $\triangle$, let $\mathcal{V}$ be its set of vertices.

Theorem 4.2. Suppose $f$ is a nonnegative function in $C^{k+1}(\Omega)$ with $0 \leq k \leq 2$. Given a triangulation $\triangle$ of $\Omega$, let $\left\{\gamma_{v}\right\}_{v \in \mathcal{V}}$ be the factors arising in Algorithm 2.1. Then

$$
\left\|f-\mathcal{I}_{P S}^{+} f\right\| \leq L_{1, \triangle}|\triangle|+K_{1}|\triangle|^{k+1}|f|_{k+1},
$$

where

$$
L_{1, \triangle}:=\max _{v \in \mathcal{V}}\left[\left(1-\gamma_{v}\right)\left(\left|f_{x}(v)\right|+\left|f_{y}(v)\right|\right) / 2\right]
$$


and $K_{1}$ is the constant in Theorem 4.1. Moreover, $L_{1, \triangle} \leq|f|_{1}$, and if $f$ is positive on $\Omega$ then $L_{1, \triangle} \rightarrow 0$ as $|\triangle| \rightarrow 0$.

Proof: Fix $f \in C^{k+1}(\Omega)$, and let $T$ be one of the triangles of $\triangle$. Then

$$
\left\|f-\mathcal{I}_{P S}^{+} f\right\|_{T} \leq\left\|f-\mathcal{I}_{P S} f\right\|_{T}+\left\|\mathcal{I}_{P S} f-\mathcal{I}_{P S}^{+} f\right\|_{T}
$$

In view of Theorem 4.1, it suffices to estimate the last term. Let $\left\{c_{i}^{o}\right\}_{i=1}^{19}$ and $\left\{c_{i}^{+}\right\}_{i=1}^{19}$ be the B-coefficients of $\left.\mathcal{I}_{P S}\right|_{T}$ and $\left.\mathcal{I}_{P S}^{+}\right|_{T}$, respectively, see Fig. 1 . Then examining the proof of Theorem 2.2 , we see that

$$
\left|c_{i}^{o}-c_{i}^{+}\right| \leq L_{1, \triangle}|\triangle|, \quad i=1, \ldots, 19
$$

Using the fact that the Bernstein basis polynomials are bounded by one, we get

$$
\left\|\mathcal{I}_{P S} f-\mathcal{I}_{P S}^{+} f\right\|_{T} \leq L_{1, \triangle}|\triangle|
$$

Since this holds for every triangle $T$, (4.2) follows.

Since $0 \leq \gamma_{v} \leq 1$ for every $v \in \mathcal{V}$, it is clear that $L_{1, \triangle} \leq|f|_{1}$. We now examine the behavior of $L_{1, \triangle}$ when $f$ is positive on $\Omega$. Let $v$ be a vertex of $\triangle$. By construction $\gamma_{v}$ is either one, or $0<\gamma_{v}<1$ with

$$
\gamma_{v}=\frac{-2 f(v)}{h_{x} f_{x}(v)+h_{y} f_{y}(v)}
$$

for some $0 \leq h_{x} \leq|\triangle|$ and $0 \leq h_{y} \leq|\triangle|$. But then

$$
\gamma_{v} \geq \frac{2 f(v)}{|\triangle|\left(\left|f_{x}(v)\right|+\left|f_{y}(v)\right|\right)}
$$

Since $f>0$, this lower bound for $\gamma_{v}$ increases as $|\triangle| \rightarrow 0$, and $\gamma_{v}$ eventually becomes one for sufficiently small $|\triangle|$. It follows that

$$
L_{1, \triangle} \leq \max \left\{0, \max _{v \in \Omega}\left(\frac{\left|f_{x}(v)\right|+\left|f_{y}(v)\right|}{2}-\frac{f(v)}{|\triangle|}\right)\right\}
$$

By the continuity of $f$, there exists $\epsilon$ such that $f \geq \epsilon>0$, and it follows that $L_{1, \triangle} \rightarrow 0$ as $|\triangle| \rightarrow 0$.

This theorem shows that for all triangulations with $|\triangle|$ sufficiently small, our nonnegative Powell-Sabin interpolant provides optimal approximation order of smooth functions. 


\subsection{The Clough-Tocher Case}

The following result gives an error bound for the unrestricted Clough-Tocher interpolant $\mathcal{I}_{C T}$, see Theorem 6.8 in [7].

Theorem 4.3. For every $f \in C^{k+1}(\Omega)$ with $0 \leq k \leq 3$,

$$
\left\|D_{x}^{\alpha} D_{y}^{\beta}\left(f-\mathcal{I}_{C T} f\right)\right\| \leq K_{2}|\triangle|^{k+1-\alpha-\beta}|f|_{k+1},
$$

for all $0 \leq \alpha+\beta \leq k$. If $\Omega$ is convex, then the constant $K_{2}$ depends only on the smallest angle in $\triangle$. If $\Omega$ is not convex, then $K_{2}$ also depends on the Lipschitz constant of the boundary of $\Omega$.

We now derive an analogous error bound for our nonnegative Clough-Tocher interpolant $\mathcal{I}_{C T}^{+}$. Given a triangulation $\triangle$, let $\mathcal{V}$ be its set of vertices, and let $\mathcal{E}$ be its set of edges. Suppose that the initial gradient values $\left\{f_{i}^{x}, f_{i}^{y}\right\}_{i=1}^{n}$ at the vertices and the normal cross-derivative values $\left\{g_{e}\right\}_{e \in \mathcal{E}}$ at the midpoints $\eta_{e}$ of the edges are sampled from a smooth nonnegative function $f$. We define $\mathcal{I}_{C T}^{*} f$ as the Hermite interpolating spline in $\mathcal{S}_{3}^{1}\left(\triangle_{C T}\right)$ that satisfies

$$
\mathcal{I}_{C T}^{*} f(v)=f(v), \quad D_{x} \mathcal{I}_{C T}^{*} f(v)=\gamma_{v} D_{x} f(v), \quad \mathcal{I}_{C T}^{*} f(v)=\gamma_{v} D_{y} f(v),
$$

for all vertices $v \in \mathcal{V}$, and

$$
D_{u_{e}} \mathcal{I}_{C T}^{*} f\left(\eta_{e}\right)=D_{u_{e}} f\left(\eta_{e}\right),
$$

for all edges $e \in \mathcal{E}$. Here, $\gamma_{v}$ are the factors produced by Algorithm 3.2, $\eta_{e}$ are the midpoints of the edges, and $D_{u_{e}}$ are the directional derivatives defined in Sect. 3.1. The B-coefficients of interpolants $\mathcal{I}_{C T}^{+}$and $\mathcal{I}_{C T}^{*}$ are distinguished by a superscript plus and star, respectively.

Lemma 4.4. Suppose $f$ is a nonnegative function in $C^{k+1}(\Omega)$ with $0 \leq k \leq 3$. Given a triangulation $\triangle$ of $\Omega$, let $\triangle_{C T}$ be the associated Clough-Tocher refinement. Suppose that for each triangle $T$ and each edge $e$ of $T$, the orthogonal projection of the split point of $T$ onto $e$ lies on $e$. In addition, for each interior edge $e$ of $\triangle$, suppose (3.17) holds with strict inequality, i.e.,

$$
a>\frac{2+A(1-B)}{3 A B},
$$

with $A$ and $B$ the constants defined in Lemma 3.5, and suppose the same inequality holds for each boundary edge $e$ of $\triangle$ with

$$
A:=\min \left\{\frac{\alpha_{1}^{e}}{\alpha_{2}^{e}}, \frac{\alpha_{2}^{e}}{\alpha_{1}^{e}}\right\}, \quad \text { and } \quad B:=\min \left\{\alpha_{1}^{e}, \alpha_{2}^{e}\right\} .
$$

Then

$$
\left|D_{u_{e}}\left(\mathcal{I}_{C T}^{*}-\mathcal{I}_{C T}^{+}\right) f\left(\eta_{e}\right)\right| \leq L_{3, \triangle}
$$


where $L_{3, \triangle}$ is bounded from above by $K_{3}|f|_{1}$ with $K_{3}$ a constant depending only on the smallest angle in $\triangle_{C T}$. Moreover, if $f>0$ on $\Omega$ then $L_{3, \triangle} \rightarrow 0$ as $|\triangle| \rightarrow 0$.

Proof: Let $t_{e}:=\left\langle v_{1}^{e}, v_{2}^{e}, v_{T_{e}}\right\rangle$ be a micro-triangle in $\triangle_{C T}$ containing $e$. Let $\delta:=$ $\left(\delta_{1}, \delta_{2}, \delta_{3}\right)$ be the directional coordinates of the unit vector $u_{e}$ relative to $t_{e}$. Then the cross derivative $D_{u_{e}} f\left(\eta_{e}\right)$ can be expressed in terms of the B-coefficients of $\left.\mathcal{I}_{C T}^{*}\right|_{t_{e}}$ (cf. Fig. 2) as

$$
\begin{aligned}
D_{u_{e}} f\left(\eta_{e}\right)=D_{u_{e}} \mathcal{I}_{C T}^{*} f\left(\eta_{e}\right)=\frac{3}{4}\left[\left(\delta_{1} c_{1}^{e, *}+\delta_{2} c_{4}^{e, *}+\delta_{3} c_{13}^{e, *}\right)\right. \\
\left.+2\left(\delta_{1} c_{4}^{e, *}+\delta_{2} c_{5}^{e, *}+\delta_{3} c_{10}^{e, *}\right)+\left(\delta_{1} c_{5}^{e, *}+\delta_{2} c_{2}^{e, *}+\delta_{3} c_{14}^{e, *}\right)\right] .
\end{aligned}
$$

Since the coefficients of $\mathcal{I}_{C T}^{*}$ and $\mathcal{I}_{C T}^{+}$appearing here agree except for $c_{10}^{e, *}$ and $c_{10}^{e,+}$, we have

$$
\left|D_{u_{e}}\left(\mathcal{I}_{C T}^{*}-\mathcal{I}_{C T}^{+}\right) f\left(\eta_{e}\right)\right|=\frac{3}{2}\left|\delta_{3}\right|\left|c_{10}^{e, *}-c_{10}^{e,+}\right| .
$$

It now suffices to consider the case where $c_{10}^{e, *}$ fails to satisfy the inequality (3.7), since otherwise $c_{10}^{e, *}$ equals $c_{10}^{e,+}$. Suppose $e$ is an interior edge, and that the other triangle sharing edge $e$ is $\hat{t}_{e} \in \triangle_{C T}$. A similar argument will hold for a boundary edge. Suppose also that $c_{10}^{e, *} \leq \hat{c}_{10}^{e, *}$. In this case $c_{10}^{e,+}$ is equal to the right-hand side of (3.7), and $c_{10}^{e, *}<c_{10}^{e,+}$. Using $\delta_{1}+\delta_{2}+\delta_{3}=0,\left|\delta_{i}\right| \leq K_{5}|\triangle|^{-1}$, it follows from (4.7) and the nature of the B-coefficients that

$$
\left|\delta_{1} c_{4}^{e, *}+\delta_{2} c_{5}^{e, *}+\delta_{3} c_{10}^{e, *}\right| \leq K_{6}|f|_{1, t_{e}} .
$$

In view of our restriction on the CT-refinement, $\delta_{1}, \delta_{2} \geq 0$ or $\delta_{1}, \delta_{2} \leq 0$. Then,

$$
K_{6}|f|_{1, t_{e}} \geq\left|\delta_{1}+\delta_{2}\right| \min \left\{c_{4}^{e, *}, c_{5}^{e, *}\right\}-\left|\delta_{3}\right| c_{10}^{e, *} \geq\left|\delta_{3}\right|\left(\min \left\{c_{4}^{e, *}, c_{5}^{e, *}\right\}-c_{10}^{e, *}\right),
$$

or

$$
\left|\delta_{3}\right|\left(c_{10}^{e, *}-c_{10}^{e,+}\right) \geq-K_{6}|f|_{1, t_{e}}+\left|\delta_{3}\right|\left(\min \left\{c_{4}^{e, *}, c_{5}^{e, *}\right\}-c_{10}^{e,+}\right) .
$$

Using $\left|\delta_{3}\right| \geq K_{7}|\triangle|^{-1}$ and our assumption that $c_{10}^{e, *}<c_{10}^{e,+}$, we find that

$$
\left|\delta_{3}\right|\left|c_{10}^{e, *}-c_{10}^{e,+}\right| \leq \max \left\{0, K_{6}|f|_{1, t_{e}}-K_{7}|\triangle|^{-1}\left(\min \left\{c_{4}^{e, *}, c_{5}^{e, *}\right\}-c_{10}^{e,+}\right)\right\} .
$$

Defining

$$
M:=\frac{m}{2} A\left[\left(1+\frac{1}{3 a}\right) B-\frac{1}{3 a}\right],
$$

where $m$ is as in Lemma 3.1, (3.6), (3.7) and (3.10) imply $\min \left\{c_{4}^{e, *}, c_{5}^{e, *}\right\} \geq-m /(3 a)$ and $c_{10}^{e,+} \leq-M$. Thus,

$$
\left|\delta_{3}\right|\left|c_{10}^{e, *}-c_{10}^{e,+}\right| \leq \max \left\{0, K_{6}|f|_{1, t_{e}}-K_{7}|\Delta|^{-1}\left(M-\frac{m}{3 a}\right)\right\} .
$$

Since $m \geq 0$, using (4.5) we have $M-m /(3 a) \geq 0$. Thus, we can bound (4.9) from above by $K_{6}|f|_{1}$. If $f>0$ then $m>0$, and using (4.5) we get $M-m /(3 a) \geq \epsilon>0$. It follows that the right hand side of (4.9) decreases to 0 as $|\triangle| \rightarrow 0$.

The restriction on the CT-refinement in Lemma 4.4 is satisfied when the split points are chosen to be the incenters. This restriction is stronger than the one imposed in Theorem 3.6. 
Theorem 4.5. Suppose $f$ is a nonnegative function in $C^{k+1}(\Omega)$ with $0 \leq k \leq 3$. Given a triangulation $\triangle$ of $\Omega$, let $\triangle_{C T}$ be the associated Clough-Tocher refinement. Suppose that for each triangle $T$ and each edge $e$ of $T$, the orthogonal projection of the split point of $T$ onto $e$ lies on $e$, and suppose for each edge of $\triangle$, we choose $a_{i j}$ to satisfy (3.15) and (4.5). Then

$$
\left\|f-\mathcal{I}_{C T}^{+} f\right\| \leq L_{4, \triangle}|\triangle|+K_{2}|\triangle|^{k+1}|f|_{k+1},
$$

where $K_{2}$ is the constant in Theorem 4.3, and $L_{4, \triangle} \leq K_{4}|f|_{1}$ with $K_{4}$ a constant depending only on the smallest angle in $\triangle_{C T}$. Moreover, if $f$ is positive on $\Omega$ then $L_{4, \triangle} \rightarrow 0$ as $|\triangle| \rightarrow 0$.

Proof: By the triangle inequality,

$$
\left\|f-\mathcal{I}_{C T}^{+} f\right\| \leq\left\|f-\mathcal{I}_{C T} f\right\|+\left\|\mathcal{I}_{C T} f-\mathcal{I}_{C T}^{*} f\right\|+\left\|\mathcal{I}_{C T}^{*} f-\mathcal{I}_{C T}^{+} f\right\|
$$

The first term on the right-hand side can be bounded by $K_{2}|\triangle|^{k+1}|f|_{k+1}$ using Theorem 4.3. By an argument similar to the one in the proof of Theorem 4.2, we find that the second term can be bounded by $L_{1, \triangle}|\triangle|$.

Let $T$ be one of the triangles of $\triangle$. Let $\left\{c_{i}^{*}\right\}_{i=1}^{19}$ and $\left\{c_{i}^{+}\right\}_{i=1}^{19}$ be the Bcoefficients of $\left.\mathcal{I}_{C T}^{*}\right|_{T}$ and $\left.\mathcal{I}_{C T}^{+}\right|_{T}$, respectively, see Fig. 2. Combining (4.6), (4.8) and $\left|\delta_{3}\right| \geq K_{7}|\triangle|^{-1}$, we get

$$
\left|c_{i}^{*}-c_{i}^{+}\right| \leq \frac{L_{3, \triangle}}{K_{7}}|\triangle|, \quad i=1, \ldots, 19
$$

Using the fact that the Bernstein basis polynomials are bounded by one, we get

$$
\left\|\mathcal{I}_{C T}^{*} f-\mathcal{I}_{C T}^{+} f\right\|_{T} \leq \frac{L_{3, \triangle}}{K_{7}}|\triangle|
$$

Since this holds for every triangle $T,(4.10)$ follows.

If we interpolate slightly different cross-derivatives, we can weaken the restriction on the CT-refinement in Theorem 4.5 to the restriction used in Theorem 3.6. For each interior edge $e$ of $\triangle$, let $w_{e}$ be a unit vector parallel to the line joining the split points of the two triangles sharing $e$. For every boundary edge $e$ of $\triangle$, let $w_{e}$ be the unit vector parallel to the line joining the midpoint of $e$ and the split point of the triangle containing $e$. We now take $\left\{g_{e}\right\}_{e \in \mathcal{E}}$, such that the (unrestricted) Hermite interpolating Clough-Tocher spline $\mathcal{J}_{C T} f$ satisfies

$$
D_{w_{e}} \mathcal{J}_{C T} f\left(\eta_{e}\right)=D_{w_{e}} f\left(\eta_{e}\right)
$$

for all edges $e \in \mathcal{E}$. Let $\mathcal{J}_{C T}^{+} f$ be the corresponding nonnegative Clough-Tocher spline. The next theorem can be proved in a similar way as Theorem 4.5. 
Theorem 4.6. Suppose $f$ is a nonnegative function in $C^{k+1}(\Omega)$ with $0 \leq k \leq 3$. Given a triangulation $\triangle$ of $\Omega$, let $\triangle_{C T}$ be the associated Clough-Tocher refinement such that for each interior edge $e$, the line joining the split points of the two adjoining triangles of $\triangle$ intersect $e$. Suppose for each edge of $\triangle$, we choose $a_{i j}$ to satisfy (3.15) and (4.5). Then

$$
\left\|f-\mathcal{J}_{C T}^{+} f\right\| \leq L_{4, \triangle}|\triangle|+K_{2}|\triangle|^{k+1}|f|_{k+1},
$$

where $K_{2}$ is the constant in the analog of Theorem 4.3 for $\mathcal{J}_{C T} f$, and $L_{4, \triangle} \leq K_{4}|f|_{1}$ with a constant $K_{4}$ depending only on the smallest angle in $\triangle_{C T}$. Moreover, if $f$ is positive on $\Omega$, then $L_{4, \triangle} \rightarrow 0$ as $|\triangle| \rightarrow 0$.

When the diameter of the triangulation is small enough, we get optimal approximation order for $\mathcal{I}_{C T}^{+} f$ and $\mathcal{J}_{C T}^{+} f$.

\section{$\S 5$. Range Restricted Interpolation}

In this section we discuss how the above methods can be adapted to deal with interpolation of scattered data with range restrictions.

\subsection{The case of a one-sided constraint}

Suppose we want to construct a spline $s$ interpolating given values $\left\{f_{i}\right\}_{i=1}^{n}$ at scattered data points $\left\{v_{i}\right\}_{i=1}^{n}$ in a domain $\Omega$, where $s$ should also lie above a given surface $s_{L}$ defined on $\Omega$. In general this can be a difficult problem, but if $s_{L}$ is taken to be a spline from the same space as $s$, it is straightforward. Indeed, in this case, we can take $s=s_{L}+s_{d}$, where $s_{d}$ is a nonnegative spline interpolating the values $\left\{f_{i}-s_{L}\left(x_{i}, y_{i}\right)\right\}_{i=1}^{n}$ at the data points. We can use this approach with both of the spline spaces discussed above. The same idea can be used to construct an interpolating spline lying below a given surface $s_{U}$.

\subsection{The case of two-sided constraints}

In this section we consider interpolation of scattered data with a spline satisfying $L \leq s(x, y) \leq U$ for all $(x, y) \in \Omega$, where $L<U$ are given constants. This problem can be solved with minor modifications of the above methods.

For the Powell-Sabin method, we simply replace step 2) in Algorithm 2.1 by the following:

2a) for $j=1, \ldots, n_{i}^{P S}$ : if $d_{i j}<0$, set $\gamma_{i}=\min \left\{\gamma_{i}, 2\left(L-f_{i}\right) / d_{i j}\right\}$,

2b) for $j=1, \ldots, n_{i}^{P S}$ : if $d_{i j}>0$, set $\gamma_{i}=\min \left\{\gamma_{i}, 2\left(U-f_{i}\right) / d_{i j}\right\}$.

The situation for the Clough-Tocher method of Sect. 3 is similar. First, in Algorithm 3.2 we must replace step 2) by

2a) for $j=1, \ldots, n_{i}$ : if $d_{i j}<0$, set $\gamma_{i}=\min \left\{\gamma_{i},\left[3\left(L-f_{i}\right)+\left(L-m_{i j}\right) / a_{i j}\right] / d_{i j}\right\}$,

2b) for $j=1, \ldots, n_{i}$ : if $d_{i j}>0$, set $\gamma_{i}=\min \left\{\gamma_{i},\left[3\left(U-f_{i}\right)+\left(U-M_{i j}\right) / a_{i j}\right] / d_{i j}\right\}$, 
where for each edge $e_{i j}$ attached to $v_{i}, m_{i j}$ is defined as before, and $M_{i j}:=\max \left\{f_{k}\right.$ : $f_{k}$ is a data value associated with a vertex of a triangle containing $\left.e_{i j}\right\}$.

We also have to adjust Algorithm 3.4. What we need now is to make the coefficients of the form $c_{10}^{e}$ satisfy a modified form of (3.7), i.e.,

$$
\begin{gathered}
c_{10}^{e} \geq L-\frac{1}{2} \min \left\{\frac{\alpha_{1}^{e}}{\alpha_{2}^{e}}, \frac{\alpha_{2}^{e}}{\alpha_{1}^{e}}\right\} \min \left\{c_{13}^{e}-L, c_{14}^{e}-L\right\}, \\
c_{10}^{e} \leq U+\frac{1}{2} \min \left\{\frac{\alpha_{1}^{e}}{\alpha_{2}^{e}}, \frac{\alpha_{2}^{e}}{\alpha_{1}^{e}}\right\} \min \left\{U-c_{13}^{e}, U-c_{14}^{e}\right\},
\end{gathered}
$$

with analogous adjustments on the conditions for $\hat{c}_{10}^{e}$.

\section{$\S 6$. Numerical Examples}

In this section we give some examples to illustrate the performance of our algorithms.

Example 6.1. Consider the test function

$$
f(x, y)=\left(x^{2}-1\right)^{2}\left(y^{2}-1\right)^{2}
$$

on the domain $\Omega:=[-1.5,1.5] \times[-1.5,1.5]$.

Discussion: Let $\left\{v_{i}\right\}_{i=1}^{n}$ be the set of 25 data points shown in Fig. 3. Let $\triangle$ be the corresponding Delaunay triangulation, and let $\triangle_{C T}$ be its Clough-Tocher refinement as shown in the figure. In Fig. 4 (left) we show a plot of the unrestricted Hermite interpolating spline $s_{u} \in \mathcal{S}_{3}^{1}\left(\triangle_{C T}\right)$ with data sampled from the function f. Fig. 4 (right) shows a plot of the nonnegative spline $s_{+} \in \mathcal{S}_{3}^{1}\left(\triangle_{C T}\right)$ produced by the method of Sect. 3. We can see clearly that $s_{u}$ takes on negative values (its minimum is -1.02), while $s_{+}$remains nonnegative. Moreover, $\left\|f-s_{+}\right\|=.98$ while $\left\|f-s_{u}\right\|=1.03$, measured in the maximum norm on a $400 \times 400$ grid. Thus, our restricted spline actually provides a slightly better fit than the unrestricted one.

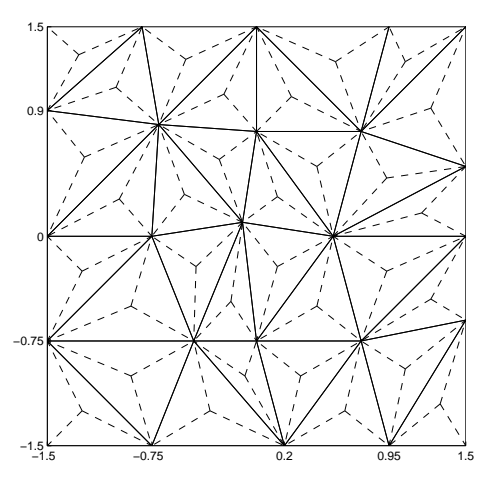

Fig. 3. A Delaunay triangulation of 25 points in the domain $\Omega$ of Example 6.1, and the corresponding CT-refinement (indicated with dashed lines). 

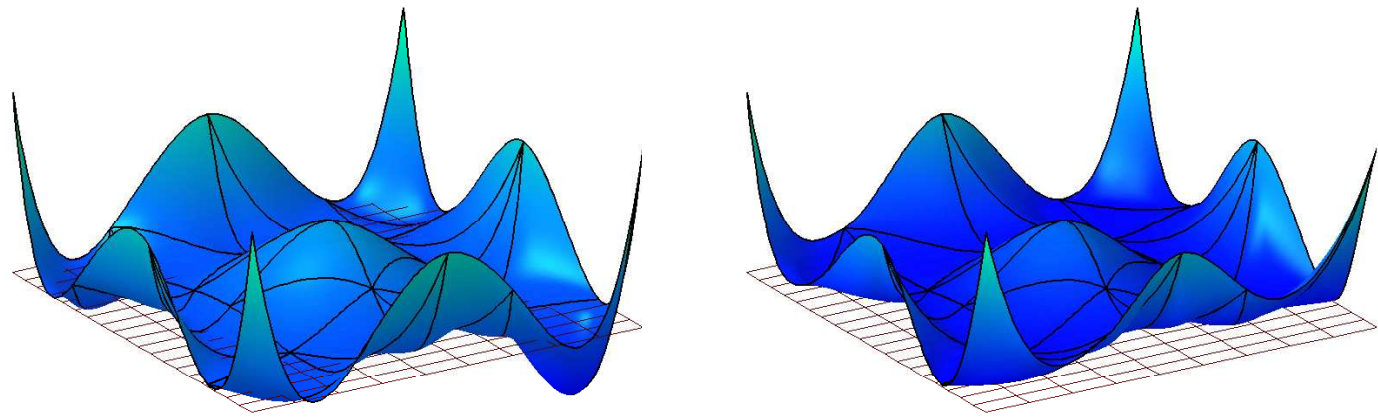

Fig. 4. The Hermite Clough-Tocher interpolant of the function in Example 6.1 and our nonnegative Clough-Tocher interpolant based on the triangulation in Fig. 3. The plane $z=0$ is shown as a grid.

To understand better how our nonnegative spline interpolants compare to their unrestricted counterparts, we now compare errors on a sequence of nested triangulations.

Example 6.2. Let $f$ and $\Omega$ be as in Example 6.1. Let $\triangle_{0}, \triangle_{1}, \ldots$ be a nested sequence of type- 1 triangulations of $\Omega$, where $\triangle_{0}$ consists of 8 triangles, and the successive triangulations are obtained by uniform dyadic refinement.

Discussion: For each $i=0,1, \ldots$, the number of triangles in $\triangle_{i}$ is easily seen to be $8 * 4^{i}$. We compare our methods with the standard Hermite interpolants for both of the spline spaces $\mathcal{S}_{2}^{1}\left(\triangle_{P S}\right)$ and $\mathcal{S}_{3}^{1}\left(\triangle_{C T}\right)$, where in all cases we use exact derivatives of the test function as data. Tab. 1 shows the results for levels $i=0, \ldots, 6$, where we have reported the maximum errors on a $400 \times 400$ grid for the unrestricted spline interpolants along with the errors for our nonnegative interpolants. We also give the minimum values of the unrestricted spline interpolants on $\Omega$.

The table shows that for the coarser grids, the unrestricted splines fail to be nonnegative by substantial amounts, but for the finer grids they become almost nonnegative. This is to be expected since for the finer grids, the splines are very close fits to the true function. It also shows that for the coarser grids, our nonnegative splines actually produce smaller errors than the unrestricted splines. The errors for the unrestricted Powell splines are of order $\mathcal{O}\left(|\triangle|^{3}\right)$, while for the unrestricted Clough-Tocher splines they are of order $\mathcal{O}\left(\mid \triangle^{4}\right)$. This matches with the known error bounds for these spaces, see Theorems 4.1 and 4.3. The order of approximation for both of our nonnegative-preserving methods seems to be $\mathcal{O}\left(|\triangle|^{2}\right)$.

We now examine the behavior of quantity $L_{1, \triangle}$ in Theorem 4.2 for the function 


\begin{tabular}{|c|rr|r|rr|r|}
\hline \multirow{2}{*}{ level } & \multicolumn{3}{|c|}{ Powell-Sabin } & \multicolumn{3}{c|}{ Clough-Tocher } \\
\cline { 2 - 7 } & \multicolumn{2}{|c|}{ unrestricted } & nonnegative & \multicolumn{2}{c|}{ unrestricted } & nonnegative \\
\cline { 2 - 7 } & min & error & error & min & error & error \\
\hline 0 & -3.76 & 3.76 & 1.47 & -3.17 & 3.17 & 1.67 \\
2 & -1.17 & 1.21 & $5.99 \mathrm{e}-1$ & -1.02 & 1.03 & $8.10 \mathrm{e}-1$ \\
3 & $-6.89 \mathrm{e}-2$ & $1.98 \mathrm{e}-1$ & $2.02 \mathrm{e}-1$ & $-4.86 \mathrm{e}-2$ & $1.41 \mathrm{e}-1$ & $1.23 \mathrm{e}-1$ \\
4 & $-8.94 \mathrm{e}-3$ & $2.54 \mathrm{e}-2$ & $2.54 \mathrm{e}-2$ & $-4.40 \mathrm{e}-3$ & $1.25 \mathrm{e}-2$ & $1.90 \mathrm{e}-2$ \\
5 & $-9.07 \mathrm{e}-4$ & $3.04 \mathrm{e}-3$ & $3.27 \mathrm{e}-3$ & $-2.17 \mathrm{e}-4$ & $9.24 \mathrm{e}-4$ & $3.14 \mathrm{e}-3$ \\
6 & $-4.70 \mathrm{e}-5$ & $3.73 \mathrm{e}-4$ & $8.60 \mathrm{e}-4$ & $-5.19 \mathrm{e}-6$ & $6.26 \mathrm{e}-5$ & $6.85 \mathrm{e}-4$ \\
& $-3.17 \mathrm{e}-7$ & $4.61 \mathrm{e}-5$ & $2.02 \mathrm{e}-4$ & $-2.96 \mathrm{e}-7$ & $3.45 \mathrm{e}-6$ & $1.44 \mathrm{e}-4$ \\
\hline
\end{tabular}

Tab. 1. Hermite interpolation versus nonnegativity preserving interpolation for Example 6.2, using Powell-Sabin and Clough-Tocher splines. The first column denotes the number of mesh refinement levels. For each level, the minimal value and the error of the unrestricted splines are shown, together with the error of the corresponding nonnegativity preserving splines. All errors are measured in the $L_{\infty}$-norm.

$f$ in (6.1). Using the upper bound (4.3), we have

$$
\begin{aligned}
L_{1, \triangle} & \leq \max \left\{0, \max _{v \in \Omega}\left(\max \left\{\left|f_{x}(v)\right|,\left|f_{y}(v)\right|\right\}-\frac{f(v)}{|\triangle|}\right)\right\} \\
& \leq \max _{(x, y) \in \Omega}\left(y^{2}-1\right)^{2}\left(4|x|\left|x^{2}-1\right|-\frac{\left(x^{2}-1\right)^{2}}{|\triangle|}\right) \\
& \leq \max _{x \in[-1.5,1.5]} \frac{25}{16}\left(6\left|x^{2}-1\right|-\frac{\left(x^{2}-1\right)^{2}}{|\triangle|}\right) .
\end{aligned}
$$

Let $|\triangle| \leq 1 / 3$. Then the maximum value is obtained at the points $\tilde{x}= \pm \sqrt{1 \pm 3|\triangle|}$, and we get

$$
L_{1, \triangle} \leq \frac{225}{16}|\triangle|
$$

which explains the quadratic convergence of the error.

Example 6.3. Consider the positive test function

$$
f(x, y)=\left(x^{2}-1\right)^{2}\left(y^{2}-1\right)^{2}+0.001
$$

on the domain $\Omega:=[-1.5,1.5] \times[-1.5,1.5]$. Let $\triangle_{0}, \triangle_{1}, \ldots$ be the nested sequence of type-1 triangulations of $\Omega$ in Example 6.2.

Discussion: We compare our nonnegativity preserving methods with the standard Hermite interpolants for both of the spline spaces $\mathcal{S}_{2}^{1}\left(\triangle_{P S}\right)$ and $\mathcal{S}_{3}^{1}\left(\triangle_{C T}\right)$, where in all cases we use exact derivatives of the test function as data. Tab. 2 shows the results for levels $i=0, \ldots, 6$, where the maximum errors are given for the unrestricted spline interpolants along with the errors for our nonnegative interpolants. 


\begin{tabular}{|c|rr|r|rr|r|}
\hline \multirow{2}{*}{ level } & \multicolumn{3}{|c|}{ Powell-Sabin } & \multicolumn{3}{c|}{ Clough-Tocher } \\
\cline { 2 - 7 } & \multicolumn{2}{|c|}{ unrestricted } & nonnegative & \multicolumn{2}{c|}{ unrestricted } & nonnegative \\
\cline { 2 - 7 } & min & error & error & min & error & error \\
\hline 0 & -3.76 & 3.76 & 1.47 & -3.17 & 3.17 & 1.67 \\
2 & -1.17 & 1.21 & $5.98 \mathrm{e}-1$ & -1.02 & 1.03 & $8.10 \mathrm{e}-1$ \\
3 & $-6.79 \mathrm{e}-2$ & $1.98 \mathrm{e}-1$ & $2.01 \mathrm{e}-1$ & $-4.76 \mathrm{e}-2$ & $1.41 \mathrm{e}-1$ & $1.22 \mathrm{e}-1$ \\
4 & $-7.94 \mathrm{e}-3$ & $2.54 \mathrm{e}-2$ & $2.54 \mathrm{e}-2$ & $-3.40 \mathrm{e}-3$ & $1.25 \mathrm{e}-2$ & $1.83 \mathrm{e}-2$ \\
5 & $9.32 \mathrm{e}-5$ & $3.04 \mathrm{e}-3$ & $3.04 \mathrm{e}-3$ & $7.83 \mathrm{e}-4$ & $9.24 \mathrm{e}-4$ & $2.43 \mathrm{e}-3$ \\
6 & $9.53 \mathrm{e}-4$ & $3.73 \mathrm{e}-4$ & $3.73 \mathrm{e}-4$ & $9.95 \mathrm{e}-4$ & $6.26 \mathrm{e}-5$ & $1.37 \mathrm{e}-4$ \\
& $1.00 \mathrm{e}-3$ & $4.61 \mathrm{e}-5$ & $4.61 \mathrm{e}-5$ & $1.00 \mathrm{e}-3$ & $3.45 \mathrm{e}-6$ & $3.45 \mathrm{e}-6$ \\
\hline
\end{tabular}

Tab. 2. Hermite interpolation versus nonnegativity preserving interpolation for Example 6.3, using Powell-Sabin and Clough-Tocher splines. The first column denotes the number of mesh refinement levels. For each level, the minimal value and the error of the unrestricted splines are shown, together with the error of the corresponding nonnegativity preserving splines. All errors are measured in the $L_{\infty}$-norm.

The minimum values of the unrestricted spline interpolants on $\Omega$ are also shown in the table, and we see that they are positive on $\triangle_{i}$ with $i \geq 4$. On the coarser grids our nonnegative splines actually produce smaller errors than the unrestricted splines, and for levels $i \geq 6$ the corresponding restricted and unrestricted splines are identical.

For $|\triangle| \leq 1 / 3$, the quantity $L_{1, \triangle}$ in Theorem 4.2 for function $f$ in (6.2) satisfies

$$
L_{1, \triangle} \leq \max \left\{0,\left(\frac{225}{16}-\frac{1}{1000|\triangle|^{2}}\right)|\triangle|\right\},
$$

which reveals that the approximation order is better than quadratic, and that the restricted and unrestricted Powell-Sabin spline interpolants are identical when $|\triangle| \leq \sqrt{10} / 375$.

Our next example deals with range-restricted interpolation.

Example 6.4. Consider the function

$$
f(x, y)= \begin{cases}1, & y-x>\frac{1}{2}, \\ 2(y-x), & 0 \leq y-x \leq \frac{1}{2}, \\ \frac{1}{2} \cos \left(4 \pi \sqrt{\left(x-\frac{3}{2}\right)^{2}+\left(y-\frac{1}{2}\right)^{2}}\right)+\frac{1}{2}, & \left(x-\frac{3}{2}\right)^{2}+\left(y-\frac{1}{2}\right)^{2} \leq \frac{1}{16}, \\ 0, & \text { otherwise, }\end{cases}
$$

on the domain $\Omega:=[0,2] \times[0,1]$.

Discussion: This function is taken from [6], and is bounded below by the plane $z=0$ and bounded above by the plane $z=1$. We will use again a nested sequence of triangulations $\triangle_{0}, \triangle_{1}, \ldots$, where $\triangle_{0}$ is a type- 1 triangulation consisting 
of 8 triangles, and the successive triangulations are obtained by uniform dyadic refinement.

We compare our range-restricted methods with the standard Hermite interpolants for both of the spline spaces $\mathcal{S}_{2}^{1}\left(\triangle_{P S}\right)$ and $\mathcal{S}_{3}^{1}\left(\triangle_{C T}\right)$, where in all cases we use exact derivatives of the test function as data. We require our range-restricted spline interpolants to satisfy $0 \leq s(x, y) \leq 1$ throughout $\Omega$. Tab. 3 shows the results for levels $i=0, \ldots, 6$, where we have reported the maximum errors for the unrestricted spline interpolants along with the errors using our range-restricted methods. We also give the minimum and maximum values of the unrestricted spline interpolants on $\Omega$.

\begin{tabular}{|c|rrr|r|rrr|r|}
\hline \multirow{4}{*}{ level } & \multicolumn{4}{|c|}{ Powell-Sabin } & \multicolumn{4}{c|}{ Clough-Tocher } \\
\cline { 2 - 9 } & \multicolumn{2}{|c|}{ unrestricted } & restricted & \multicolumn{2}{c|}{ unrestricted } & restricted \\
\cline { 2 - 9 } & min & max & error & error & min & max & error & error \\
\hline 0 & $-3.33 \mathrm{e}-1$ & 1.167 & 1.0 & 1.0 & $-3.01 \mathrm{e}-1$ & 1.148 & 1.0 & 1.0 \\
2 & $-1.66 \mathrm{e}-1$ & 1.167 & $6.95 \mathrm{e}-1$ & $6.95 \mathrm{e}-1$ & $-1.50 \mathrm{e}-1$ & 1.148 & $6.23 \mathrm{e}-1$ & $6.23 \mathrm{e}-1$ \\
3 & $-8.33 \mathrm{e}-2$ & 1.083 & $1.56 \mathrm{e}-1$ & $1.56 \mathrm{e}-1$ & $-7.52 \mathrm{e}-2$ & 1.075 & $1.29 \mathrm{e}-1$ & $1.29 \mathrm{e}-1$ \\
4 & $-4.16 \mathrm{e}-2$ & 1.042 & $4.16 \mathrm{e}-2$ & $4.17 \mathrm{e}-2$ & $-3.76 \mathrm{e}-2$ & 1.038 & $3.76 \mathrm{e}-2$ & $3.76 \mathrm{e}-2$ \\
5 & $-2.08 \mathrm{e}-2$ & 1.021 & $2.08 \mathrm{e}-2$ & $2.08 \mathrm{e}-2$ & $-1.88 \mathrm{e}-2$ & 1.019 & $1.88 \mathrm{e}-2$ & $1.88 \mathrm{e}-2$ \\
6 & $-1.04 \mathrm{e}-2$ & 1.010 & $1.04 \mathrm{e}-2$ & $1.04 \mathrm{e}-2$ & $-9.38 \mathrm{e}-3$ & 1.009 & $9.38 \mathrm{e}-3$ & $9.39 \mathrm{e}-3$ \\
& $-5.20 \mathrm{e}-3$ & 1.005 & $5.20 \mathrm{e}-3$ & $5.20 \mathrm{e}-3$ & $-4.69 \mathrm{e}-3$ & 1.005 & $4.69 \mathrm{e}-3$ & $4.69 \mathrm{e}-3$ \\
\hline
\end{tabular}

Tab. 3. Hermite interpolation versus range-restricted interpolation for Example 6.4, using Powell-Sabin and Clough-Tocher splines. The first column denotes the number of mesh refinement levels. For each level, the minimal and maximal value of the unrestricted splines are shown, and the $L_{\infty}$-norms of the errors of the unrestricted and range-restricted splines are given.

The table shows that for the coarser grids, the unrestricted splines fail the range constraints by substantial amounts, but for the finer grids they are almost satisfied. For this example, the range-restricted splines actually have essentially the same errors as the unrestricted splines. The errors in all cases are of order $\mathcal{O}(|\triangle|)$ due to the fact that the test function is only $C^{0}$. Fig. 5 shows both the unrestricted Hermite interpolant based on Clough-Tocher splines on $\triangle_{1}$ and the corresponding range-restricted interpolant.

\section{$\S 7$. Remarks}

Remark 1. The problem of estimating the gradients of an unknown function given only values of $f$ at a set of scattered points $\left\{v_{i}\right\}_{i=1}^{n}$ has been heavily studied in the literature. Typical methods involve doing local least squares with low degree polynomials or radial basis functions. For some specific methods and further references, see $[4,14]$. 

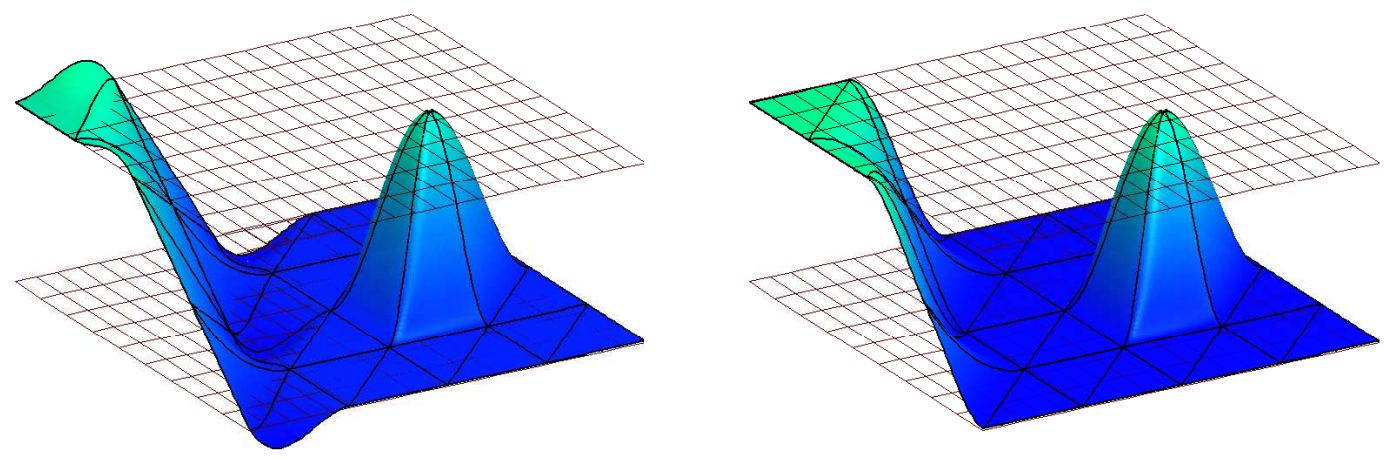

Fig. 5. Hermite interpolation versus nonnegativity preserving interpolation to function (6.3) using Clough-Tocher splines on the type-1 triangulation $\Delta_{1}$ of $\Omega$. The planes $z=0$ and $z=1$ are shown as grids.

Remark 2. The same methods for estimating gradients at the $\left\{v_{i}\right\}_{i=1}^{n}$ can be used to estimate cross derivatives at points on the edges of a triangulation when needed. Alternatively, we can assume the cross derivatives vary linearly along each edge, in which case the needed values can be computed from the gradients at the endpoints of the edges.

Remark 3. A complete set of necessary and sufficient nonnegativity conditions for bivariate quadratic polynomials was established in $[2,10]$. However, because of their complicated nonlinear structure, these conditions are rather difficult to use in practice. A set of necessary and sufficient conditions for bivariate cubic polynomials is not derived yet. A sufficient criterion for nonnegativity is to require that all Bcoefficients of the polynomial are nonnegative. Weaker sufficient conditions were given in $[1,11]$.

Remark 4. In carrying out Algorithm 2.1, it suffices to perform step 2) only for edges of $\triangle_{P S}$ that are not interior edges of $\triangle$. Making $c_{5}$ (and the analogous coefficient in the neighboring triangle) nonnegative insures $c_{4} \geq 0$.

Remark 5. The method of Sect. 2 implicitly solves a linear programming problem to determine the parameters $\gamma_{i}$ :

$$
\begin{aligned}
\max \gamma_{i}, & \text { subject to } \\
& 0 \leq \gamma_{i} \leq 1, \\
& 2 f_{i}+\gamma_{i} d_{i j} \geq 0, \quad \text { for } j=1, \ldots, n_{i}^{P S} .
\end{aligned}
$$

For some related optimization strategies, see [9]. 
Remark 6. It is easy to construct a simple example to show that Lemma 3.5 does not hold for any choice of $a$ on some triangulations containing a negative $\beta_{1}^{e}$ or a negative $\beta_{2}^{e}$. Suppose that $t_{e}:=\left\langle v_{1}^{e}, v_{2}^{e}, v_{T_{e}}\right\rangle$ and $\hat{t}_{e}:=\left\langle v_{2}^{e}, v_{1}^{e}, v_{\hat{T}_{e}}\right\rangle$ are two micro-triangles of a CT-refinement $\triangle_{C T}$ sharing an edge $e:=\left\langle v_{1}^{e}, v_{2}^{e}\right\rangle$, and that the split points $v_{T_{e}}$ and $v_{\hat{T}_{e}}$ are taken to be barycenters of macro-triangles $T_{e}$ and $\hat{T}_{e}$. Suppose in addition that the shape of these triangles is such that $\beta_{1}^{e}, \beta_{3}^{e}<0$, while $\beta_{2}^{e} \geq 0$. We choose the values at $v_{1}^{e}$ and $v_{2}^{e}$ so that $c_{2}^{e}>0$ and $c_{1}^{e}>-c_{2}^{e}\left(\left(1-\beta_{3}^{e}\right) / 2+\beta_{2}^{e}\right) / \beta_{1}^{e}$. One can easily see that in this case $c_{1}^{e}>c_{2}^{e}$, since $\beta_{2}^{e}=1+\left|\beta_{1}^{e}\right|+\left|\beta_{3}^{e}\right|$ implies that $-\left(\left(1-\beta_{3}^{e}\right) / 2+\beta_{2}^{e}\right) / \beta_{1}^{e} \geq 1-3 /\left(2 \beta_{1}^{e}\right) \geq 1$. Then, with zero gradients at both $v_{1}^{e}$ and $v_{2}^{e}$, we get $c_{13}^{e}=\hat{c}_{13}^{e}=c_{4}^{e}=c_{1}^{e}$ and $c_{14}^{e}=\hat{c}_{14}^{e}=c_{5}^{e}=c_{2}^{e}$. This gives

$$
\begin{aligned}
I & =\frac{1}{2} \min \left\{c_{1}^{e}, c_{2}^{e}\right\}+\beta_{1}^{e} c_{1}^{e}+\beta_{2}^{e} c_{2}^{e}-\frac{\beta_{3}^{e}}{2} \min \left\{c_{1}^{e}, c_{2}^{e}\right\} \\
& =\frac{1}{2}\left(1-\beta_{3}^{e}\right) c_{2}^{e}+\beta_{1}^{e} c_{1}^{e}+\beta_{2}^{e} c_{2}^{e} \\
& <\frac{1}{2}\left(1-\beta_{3}^{e}\right) c_{2}^{e}-\left(\frac{1}{2}\left(1-\beta_{3}^{e}\right)+\beta_{2}^{e}\right) c_{2}^{e}+\beta_{2}^{e} c_{2}^{e}=0 .
\end{aligned}
$$

Remark 7. The nonnegativity restriction imposed on our Powell-Sabin spline is a sufficient but not a necessary condition, see Remark 3. Therefore the method described in Sect. 2.2 is not able to reproduce all nonnegative quadratic polynomials. However, any nonnegative linear polynomial can be exactly represented. It is easy to see that in this case Algorithm 2.1 produces $\gamma_{i}=1$ for all $i$, since, e.g., for any point $v_{i j}$ on triangle $T:=\left\langle v_{i}, v_{k}, v_{l}\right\rangle$, we have $2 f_{i}+d_{i j} \geq f_{i}+\min \left\{f_{i}, f_{k}, f_{l}\right\} \geq 0$, or $-2 f_{i} / d_{i j} \geq 1$ if $d_{i j}<0$. In a similar way one can check that the method based on the Clough-Tocher macro-element can exactly reproduce any nonnegative linear polynomial, but not all quadratic or cubic polynomials.

Remark 8. In [6] it was suggested to choose each parameter $a_{i j}=8 / 3$. This is a valid choice in the case where all CT-split points are allowed to be taken as barycenters. However, this is not generally applicable, see Remark 6 .

Remark 9. In [16] the author proposed a global optimization strategy to obtain nonnegativity preserving interpolants based on reduced Clough-Tocher macroelements. All B-coefficients of such interpolating spline are required to be nonnegative. In this approach the same restriction on the CT-split points is imposed as assumed in Theorem 4.5, which is stronger than the restriction in Theorems 3.6 and 4.6.

Remark 10. If one already has an implementation of a Hermite interpolation method for Powell-Sabin or Clough-Tocher macro-elements, it can be adjusted in a straightforward way to produce our nonnegativity preserving interpolatnt. Our methods can also easily be integrated in more advanced data interpolation strategies, e.g., as the one proposed in [13]. 
Remark 11. The methods developed here can be easily modified to work with other classical macro-elements such as the ones based on the Powell-Sabin-12 split, the double Clough-Tocher split, and triangulated convex quadrilaterals, see Chap. 6 of [7]. For the double CT-split, this leads to somewhat weaker conditions on the parameters $a_{i j}$ that play a role in the analog of Algorithm 3.2. On triangulated convex quadrilaterals, the parameters $a_{i j}$ in the analog of Algorithm 3.2 have to be set to infinity. The same technique can also be used for the construction of nonnegative $C^{1}$ cubic macro-elements on a rectilinear partition, consisting of both triangles and convex quadrilaterals.

Remark 12. Nonnegative smoothing of noisy data using $C^{1}$ Powell-Sabin macroelements has also been studied in the literature, see $[18,19]$, but it is a global method.

Acknowledgments. Hendrik Speleers is a Postdoctoral Fellow of the Research Foundation Flanders (Belgium). Most of this work was done during a recent visit to Vanderbilt.

\section{References}

1. Chan, E. S. and B. H. Ong, Range restricted scattered data interpolation using convex combination of cubic Bézier triangles, J. Comput. Appl. Math. 136 (2001), 135-147.

2. Chang, G. Z. and T. Sederberg, Nonnegative quadratic Bézier triangular patches, Comput. Aided Geom. Design 11 (1994), 113-116.

3. Goodman, T. N. T., B. H. Ong, and K. Unsworth, Constrained interpolation using rational cubic splines, in Nurbs for Curve and Surface Design, G. E. Farin (ed), SIAM, Philadelphia, 1991, 59-74.

4. Goodman, T. N. T., H. B. Said, and L. H. T. Chang, Local derivative estimation for scattered data interpolation, Appl. Math. Comput. 68 (1995), 41-50.

5. Herrmann, M., B. Mulansky, and J. W. Schmidt, Scattered data interpolation subject to piecewise quadratic range restrictions, J. Comput. Appl. Math. 73 (1996), 209-223.

6. Kong, V. P., B. H. Ong, and K. H. Saw, Range restricted interpolation using cubic Bézier triangles, in Proc. of Computer Graphics, Visualization and Computer Vision, Science Press, Union Agency, Plzen, 2004, 125-132.

7. Lai, M. J. and L. L. Schumaker, Spline Functions on Triangulations, Cambridge University Press, Cambridge, 2007.

8. Luo, Z. and X. Peng, A $C^{1}$-rational spline in range restricted interpolation of scattered data, J. Comput. Appl. Math. 194 (2006), 255-266.

9. Mulansky, B. and J. W. Schmidt, Powell-Sabin splines in range restricted interpolation of scattered data, Computing 53 (1994), 137-154. 
10. Nadler, E., Nonnegativity of bivariate quadratic functions on a triangle, Comput. Aided Geom. Design 9 (1992), 195-205.

11. Piah, A. R. M., T. N. T. Goodman, and K. Unsworth, Positivity-preserving scattered data interpolation, in The Mathematics of Surfaces XI, R. Martin, H. Bez, and M. Sabin (eds), Springer Verlag, Berlin, 2005, 336-349.

12. Piah, A. R. M., A. Saaban, and A. A. Majid, Range restricted positivitypreserving scattered data interpolation, J. Fund. Sciences 2 (2006), 63-75.

13. Quak, E. and L. L. Schumaker, Cubic spline fitting using data dependent triangulations, Comput. Aided Geom. Design 7 (1990), 293-301.

14. Renka, R. J. and A. K. Cline, A triangle-based $C^{1}$ interpolation method, Rocky Mountain J. Math. 14 (1984), 223-238.

15. Saaban, A., A. R. M. Piah, and A. A. and Majid, Range restricted $C^{2}$ interpolant to scattered data, in Proc. of Computer Graphics, Imaging and Visualisation 2007, Bangkok, IEEE Computer Society, 2007, 183-188.

16. Schmidt, J. W., Range restricted interpolation by cubic $C^{1}$-splines on CloughTocher splits, in Advances in Multivariate Approximation, W. Haussmann, K. Jetter, M. Reimer (eds), Academie Verlag - Wiley VCH, Berlin, 1999, 253-267.

17. Schmidt, J. W. and W. Heß, Positivity of cubic polynomial on intervals and positive spline interpolation, BIT 28 (1988), 340-352.

18. Willemans, K. and P. Dierckx, Constrained surface fitting using Powell-Sabin splines, in Wavelets, Images, and Surface Fitting, P.-J. Laurent, A. LeMéhauté, and L. L. Schumaker (eds), A. K. Peters, Wellesley, MA, 1994, 511-520.

19. Willemans, K. and P. Dierckx, Nonnegative surface fitting with Powell-Sabin splines, Numer. Algorithms 9 (1995), 263-276. 